\title{
Hedgehog signaling pathway regulates the growth of ovarian cancer spheroid forming cells
}

\author{
ANASUYA RAY* , ERHONG MENG* , EDDIE REED, LALITAA. SHEVDE and RODNEY P. ROCCONI \\ University of South Alabama, Mitchell Cancer Institute, Mobile, AL, USA
}

Received December 6, 2010; Accepted January 11, 2011

DOI: 10.3892/ijo.2011.1093

\begin{abstract}
The hedgehog (Hh) pathway has been shown to be activated in numerous malignancies as well as in cancer stem cells. We sought to determine the importance of the Hh pathway in regulating growth and development of ovarian cancer spheroid-forming cells (SFCs). Ovarian cancer cell lines (ES2, TOV112D, OV90, and SKOV3) as well as a normal ovarian epithelial cell line (IOSE80) were grown in non-adherent growth conditions to form SFCs. Western blot analysis was used to determine the expression of Hh pathway proteins $\mathrm{SMOH}$, PTCH, GLI1. SFCs were treated with Hh agonists (SHH and $\mathrm{IHH}$ ) as well as an Hh inhibitor (cyclopamine) to determine changes in spheroid growth and survival. All ovarian cancer cell lines readily formed spheroids in non-adherent growth conditions while IOSE80 failed to form SFCs. Compared to IOSE80, ovarian cancer cell lines demonstrated significant activation of the Hh pathway as defined by increased expression of intranuclear GLI1. Both Hh agonists demonstrated significant increases in spheroid volume of at least 42-fold for SHH-treated cells and 46-fold for IHH-treated cells. With regard to survival, SFCs were $30-50 \%$ more resistant to cyclopamine than their corresponding monolayer cells. Despite this resistance, inhibition of the Hh pathway with cyclopamine prevented further growth of SFCs with a 10-, 5-, and 4-fold restriction in growth for ES2, SKOV3, and TOV112D, respectively. The hedgehog pathway appears to be important in regulating growth of ovarian cancer spheroid-forming cells. The activation and inhibition of this pathway demonstrates significant correlation to enhanced growth and growth restriction, respectively.
\end{abstract}

Correspondence to: Dr Rodney P. Rocconi, University of South Alabama, Mitchell Cancer Institute, 1660 Springhill Avenue, Mobile, AL 36604, USA

E-mail: rocconi@usouthal.edu

${ }^{*}$ Contributed equally

Abbreviations: OCSC, ovarian cancer stem cells; Hh, hedgehog; SHH, Sonic hedgehog; IHH, Indian hedgehog; DHH, Desert hedgehog; PTCH, patched; $\mathrm{SMOH}$, smoothened; SFCs, spheroid forming cells

Key words: ovarian cancer, spheroid forming cells, hedgehog

\section{Introduction}

Ovarian cancer is the most lethal gynecologic malignancy accounting for over 14,000 deaths annually in the USA alone (1). Although the majority of patients present with advanced disease, most will respond to cytoreductive surgery followed by first-line platinum-based combination chemotherapy. Despite this response, effective curative therapy has yet to be determined and thus most patients with advanced disease will experience a recurrence and unfortunately succumb to progressive disease (2). In this regard, novel therapeutics, including targeted biologics are currently being sought for the treatment of advanced ovarian cancer.

One hypothesis associated with this poor prognosis revolves around cancer stem cells (CSC). The origin of cancer from 'stem cell' populations was first introduced approximately 150 years ago. The conceptual basis for a stem cell origin of cancer is supported by observations that certain subpopulations of cancer cells appear to acquire stem cell-like properties such as the capacity of self-renewal, and the ability to differentiate (3). Additionally, cancer stem cells possess an innate resistance to cytotoxic agents (4). Irrespective of the success of therapy on ovarian cancer cells, if chemotherapy fails to eradicate ovarian cancer stem cells (OCSC) then the cancer will likely regenerate and predispose to a recurrence or progression of disease. As such, identifying molecular pathways involved in OCSC could lead to discoveries of targeted agents. While cancer stem cells constitute a mere $0.2-1 \%$ of the total tumor, methods have been developed to enrich them ex vivo in culture, such as growth in non-adherent growth conditions resulting in spheroid-formation. These spheroid-forming cells (SFCs) demonstrate altered cell surface and molecular markers compared to the same cells grown in monolayer and appear to enrich a more potent subpopulation of ovarian cancer cells with stem-cell like properties $(5,6)$. This enrichment of SFCs has been used in numerous studies to determine the in vitro and in vivo characteristics of cancer stem cells as well as the molecular pathways involved within.

One molecular pathway of interest is the hedgehog $(\mathrm{Hh})$ signaling pathway. Normally dormant, Hh has shown enhanced activity in numerous malignancies including ovarian cancer. Hh signaling pathway controls a variety of developmental cancer processes like carcinogenesis, tumor proliferation and differentiation, as well as playing a major role in self-renewal properties of cancer stem cells and tissue repair (7). In mammals, 
three Hh ligand proteins are present: Sonic hedgehog (SHH), Indian hedgehog (IHH) and Desert hedgehog (DHH). The canonical Hh pathway is inactive in absence of these ligands. The twelve transmembrane protein Patched (PTCH) is the common Hh receptor and functions as a tumor suppressor that inhibits smoothened (SMOH), a seven transmembrane protein from activating downstream signaling. Binding of any of these Hh ligands to PTCH, alleviates PTCH-mediated suppression of SMOH, and initiates a complex downstream signaling cascade that involves the translocation of GLI, a five-zinc finger protein transcription factor, into the nucleus $(8,9)$. As such, activated $\mathrm{Hh}$ is represented by the finding of intranuclear GLI1.

The constitutively activated Hh pathway has been found in several tumor-types, which suggests this pathway as a significant diagnostic and therapeutic target of cancer $(8,10)$. It has been also shown that a plant-derived teratogenic steroidal alkaloid, cyclopamine, inhibits the Hh pathway by antagonizing $\mathrm{SMOH}$ (11) and suppresses the growth of cancer cells both in vivo and in vitro $(12,13)$.

We conducted studies to determine the importance of $\mathrm{Hh}$ pathway in the growth and development of ovarian cancer SFCs as a surrogate for ovarian cancer stem cells.

\section{Materials and methods}

Cell culture. Ovarian cancer cell lines SKOV3, OV90, TOV112D and ES2 were purchased from American Type Culture Collection. Cells were maintained in DMEM/F12 (Invitrogen, Carlsbad, CA) supplemented with $1 \%$ sodium pyruvate (Invitrogen), $0.2 \%$ non-essential amino acids (Invitrogen), and 5\% FBS in a humidified atmosphere containing 5\% $\mathrm{CO}_{2}$ at $37^{\circ} \mathrm{C}$.

Immortalized normal ovarian surface epithelial cell line IOSE80 was obtained as a generous gift from the laboratory of Dr Nelly Auersperg (The University of British Columbia, Vancouver,Canada). The cells were grown in a 1:1 combination of two media, Medium 199 (Invitrogen) and MCDB 105 (Cell Applications Inc., San Diego, CA) with 10\% FBS in a humidified atmosphere containing $5 \% \mathrm{CO}_{2}$ at $37^{\circ} \mathrm{C}$. Ovarian cancer cell lines and normal ovarian cell lines were cultured in monolayer in the above stated conditions.

Ovarian cancer spheroid culture. Normal and cancer cell lines were cultured under conditions to enrich for cells with stem-like properties by suspending in serum-free DMEM/F12 supplemented with $5 \mu \mathrm{g} / \mathrm{ml}$ insulin (Sigma, St. Louis, MO), $20 \mathrm{ng} / \mathrm{ml}$ human recombinant epidermal growth factor (EGF; Invitrogen), $10 \mathrm{ng} / \mathrm{ml}$ basic fibroblast growth factor (bFGF; Invitrogen), and $0.4 \%$ bovine serum albumin (BSA; Sigma) in 6-well ultra low attachment plates (Corning, NY) and subsequent organization into spheres (1).

\section{Western blot experiments}

Nuclear isolation. Nuclear protein extraction was performed as described by Sadowski et al (22). A confluent monolayer of cells was washed twice in ice-cold PBS. Buffer A $(10 \mathrm{mM}$ HEPES, $10 \mathrm{mM} \mathrm{KCl}, 0.1 \mathrm{mM}$ EDTA, $200 \mu \mathrm{l}$ of 10\% IGEPAL, $1 \mathrm{mM}$ DTT and $10 \mu \mathrm{l} / \mathrm{ml}$ protease inhibitor cocktail) (Sigma cat no. P8340) was added to the plate and maintained at room temperature for $10 \mathrm{~min}$. The lysate was spun at $15,000 \mathrm{~g}$ for
$3 \mathrm{~min}$ at $4^{\circ} \mathrm{C}$. The supernatant was saved as cytosolic fraction. The pellet was suspended in buffer B (20 mM HEPES, $400 \mathrm{mM}$ $\mathrm{NaCl}, 1 \mathrm{mM}$ EDTA, $10 \%$ glycerol, $1 \mathrm{mM}$ DTT, and $10 \mu \mathrm{l} / \mathrm{ml}$ protease inhibitor cocktail and kept in a shaker for overnight at $4^{\circ} \mathrm{C}$ ). The nuclear lysate was obtained by centrifuging at $15,000 \mathrm{~g}$ for $5 \mathrm{~min}$ at $4^{\circ} \mathrm{C}$.

Isolation of whole cell protein. A confluent monolayer of cells was washed once with ice cold PBS. PBS was aspirated and $0.5 \mathrm{ml}$ of ice cold NP40 lysis buffer (1\% NP40, $150 \mathrm{mM} \mathrm{NaCl}$, $50 \mathrm{mM}$ Tris-base, and protease inhibitor cocktail $10 \mu \mathrm{l} / \mathrm{ml}$ ) was added to the plate. Cells were scraped off and transferred to a $1.5 \mathrm{ml}$ Eppendorf tube. The tube was placed on ice to ensure complete cell lysis for $1 \mathrm{~h}$. Cells were centrifuged at $14,000 \mathrm{~g}$ for $10 \mathrm{~min}$ and the supernatant was collected as the whole cell lysate.

Western blot experiments. Protein concentration was measured by using Precision Red protein assay reagent (Cytoskeleton, cat no. ADV02-A). Proteins ( $30 \mu \mathrm{g})$ were subjected to SDS-PAGE and transferred to polyvinylidene fluoride membrane $(0.2 \mu \mathrm{m})$. The membranes were blocked with 5\% nonfat dry milk in Tris-buffered saline Tween-20 (TBST) [1 M Tris (pH 7.3), 9\% $\mathrm{NaCl}$ and $0.05 \%$ Tween-20] and incubated with primary antibodies overnight at $4^{\circ} \mathrm{C}$. After washes with TBST (pH 7.3) and incubation with the respective horseradish peroxidase tagged secondary antibody, the blots were developed using SuperSignal (Pierce, Rockford, IL, USA). The GLI1 antibody (Santa Cruz Biotech, Santa Cruz, CA) was used at a final dilution of 1:100 and the SMOH antibody (LifeSpan Biosciences, Inc., Seattle, WA) and PTCH antibody (Santa Cruz Biotech) were used at a final concentration of 1:200.

Quantitative real-time PCR. Total RNA was isolated using Trizol reagent from ovarian cancer cell line monolayer and spheroid-forming cells according to the manufacturer instructions (Invitrogen Corp., Carlsbad, CA) and cDNA was prepared using High Capacity Reverse Transcriptase Kit (Applied Biosystem, Foster City, CA). Real-time PCR was performed using a Bio-Rad iQ5 real-time detection system (Bio-Rad). All reactions were done as three independent replicates. All assays were done using the TaqMan Gene Expression Assays from Applied Biosystems. Primers and probes for the TaqMan system were selected from the Applied Biosystems website [GLI1 assay ID: Hs01110766_m1, Patched (PTCH) assay ID: Hs00970980_m1, and Smoothened (SMOH) assay ID: Hs00170665_m1]. The relative expression mRNA levels of GLI1, PTCH, and SMOH were normalized to internal control glyceraldehyde-3-phosphate dehydrogenase (assay ID: Hs99999905_m1) levels $\left(2^{-\delta \delta C T}\right)$.

Treatment of spheroid-forming cells with Hh agonists. Ovarian cancer cell lines ES2, SKOV3, and TOV112D were grown as spheroids and seeded at $2.5 \times 10^{4}$ cells/well in 24-well ultra-low attachment plates. On the 3rd day, $3 \mu \mathrm{g} / \mathrm{ml}$ recombinant human SHH (R\&D Systems, Minneapolis, MN) and $3 \mu \mathrm{g} / \mathrm{ml}$ recombinant mouse IHH (R\&D Systems) were added to the wells (19). Fresh recombinant SHH and IHH were added to the well every $48 \mathrm{~h}$. Pictures and measurements were taken every two days. 
Localization of GLII by immunofluorescent staining. Cyclopamine, a Hh signaling inhibitor, has previously demonstrated antitumor properties via direct binding to $\mathrm{SMOH}$ (11-13). Considering intranuclear GLI1 confers activated Hh pathway, SKOV3 cells were evaluated for GLI1 location after cyclopamine treatment and compared to control (no treatment). Cells were grown on poly-L-lysine coated glass coverslips (BD Bioscience) for overnight, then incubated with $10 \mu \mathrm{M}$ cyclopamine for $24 \mathrm{~h}$, respectively, fixed in $4 \%$ paraformaldehyde for $15 \mathrm{~min}$ at room temperature, washed with PBS and permeabilized with $0.25 \%$ Triton X-100 (Sigma) for $10 \mathrm{~min}$. Primary antibody (Anti-human Gli1 antibody, Santa Cruz Biotechnology, Inc.) incubation followed for overnight at $4^{\circ} \mathrm{C}$. After washing thoroughly with PBS, cells were further probed with secondary antibody (anti-rabbit IgG-FITC, Sigma) for $1 \mathrm{~h}$ at room temperature. Nuclei were counterstained with diluted DAPI (Sigma) for 3 min before mounting and analyzing with microscope.

Treatment of spheroid-forming cells with Hh antagonist. Cyclopamine (Sigma) was dissolved in sterile DMSO and used at varying doses from 1 to $20 \mu \mathrm{M}$ in each ovarian cancer cell line (ES2, SKOV3, and TOV112D) in monolayer. Fresh cyclopamine was added to each cell line every $24 \mathrm{~h}$ and experiments were continued up to $72 \mathrm{~h}$ to determine the LD50 concentration for each cell line used.

The SFCs were seeded at a density $2.5 \times 10^{4}$ in 24 -well ultra-low attachment plates in triplicate. After three days, SFCs were treated with LD50 concentrations of cyclopamine as determined by results obtained with the monolayer-derived cells. Digital pictures with measurements of spheroids to determine the spheroid volume were taken every 2 days for a total of 8 days. The relative increase or decrease in spheroid volume was calculated per the formula $a^{2} \times b / a_{t}^{2} \times b_{t}$ where $a$ and $b$ are the smallest and largest diameter of the spheroid, respectively, and $a_{t}$ and $b_{t}$ are the diameters at given time point $t$ (14).

In order to determine cell survival, MTS assay was performed on day 8 by adding $100 \mu \mathrm{l} /$ well of MTS reagent in 24-well plates followed by incubation at $37^{\circ} \mathrm{C}$ incubator for at least $3 \mathrm{~h}$. Absorbance was read at $490 \mathrm{~nm}$. Viable cells were determined by trypan blue exclusion assay on day 8 of the experiment. Cyclopamine treated cells in both monolayer and SFCs were compared to normal non-treated controls.

\section{Results}

Ovarian cancer spheroid-forming cells. All ovarian cancer cell lines readily formed spheroids under non-adherent growth conditions while the normal ovarian epithelial cell line IOSE80, failed to form spheroids in the same growth conditions. This supports findings that spheroid-forming cells correlate to cancer and provides some rationale to evaluating these SFCs and their properties.

Ovarian cancer cells demonstrate enhanced activation of $\mathrm{Hh}$ pathway. Activation of the Hh pathway is initiated by the cell surface protein, smoothened which leads to the translocation of cytoplasmic GLI1 to the nucleus to function as a transcription factor. As such, intranuclear GLI1 was detected by Western

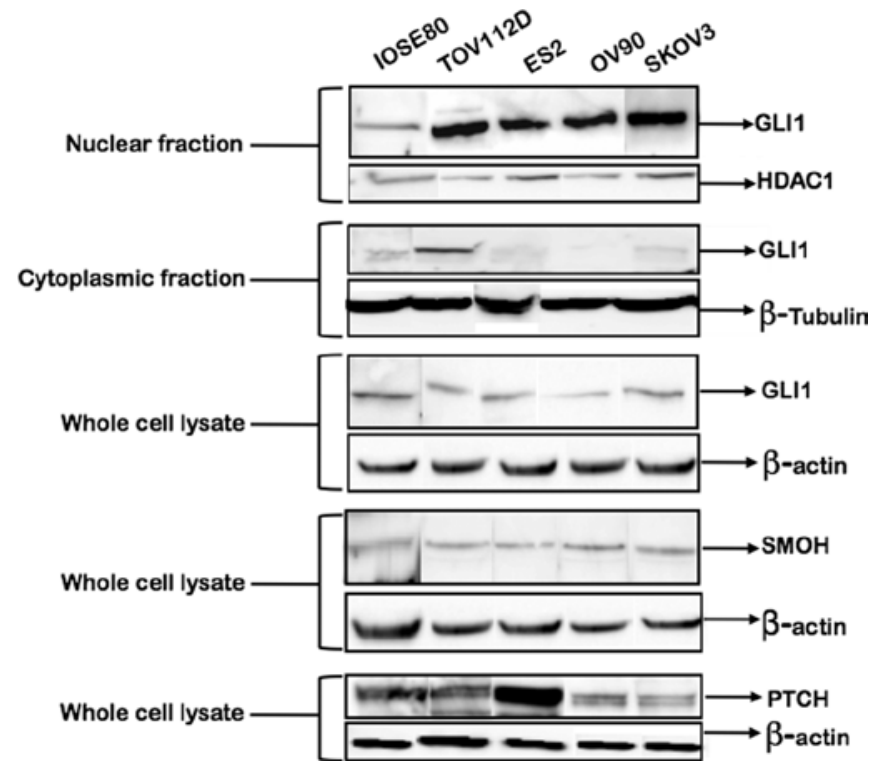

Figure 1. Ovarian cancer cells demonstrate enhanced activation of Hh pathway. Downstream effects of transmembrane Hh proteins PTCH and $\mathrm{SMOH}$ involves the translocation of cytoplasmic GLI1 to the nucleus to function as a transcription factor. As such, expression of nuclear GLI1 represents activation of the Hh pathway. Western blot experiments were performed to detect $\mathrm{Hh}$ associated proteins GLI1, SMOH, and PTCH with appropriate controls in all ovarian cancer cell lines as well as immortalized normal ovarian surface epithelial cell line (IOSE80). GLI1 was detected in lysate of all cell lines evaluated. Nuclear fraction of GLI1 was significantly expressed in all ovarian cancer cell lines (SKOV3, OV90, TOV112D, and ES2) compared to normal epithelial ovarian cells (IOSE80). Other Hh proteins (SMOH and PTCH) were expressed at varying levels in all cell lines evaluated.

blot analysis in nuclear fraction from all cell lines evaluated with increased expression in ovarian cancer cell lines (ES2, SKOV3 and TOV112D) compared to immortalized normal ovarian cell line (IOSE80). Consistent with a normally dormant innate pathway, all cell lines expressed varying levels of $\mathrm{SMOH}$ and PTCH (Fig. 1).

Real-time PCR was used to evaluate the differences in $\mathrm{Hh}$ mediators found in monolayer and SFCs of the several cell lines (ES2, SKOV3, and TOV112D) (Fig. 2). A Hh agonist, $\mathrm{SHH}$, was significantly upregulated in all SFCs compared to monolayer. As such, Hh pathway mediators are likely important in the cancer stem cell property of spheroid formation. Of note, was the correlation of up/down-regulation of GLI1 (monolayer to SFCs) to the histologic innate aggressiveness of various cell lines. In order of increasing aggressiveness: TOV112D (endometrioid) showed a significant 0.7-fold down-regulation; SKOV3 (papillary serous) significant 2.3-fold upregulation; and ES2 (clear cell) significant 7-fold upregulation.

Activated Hh pathway significantly enhances ovarian cancer spheroid growth. Spheroid-forming cells derived from ES2, SKOV3, and TOV112D cell lines were treated with Hh agonists and monitored for growth. All ovarian cancer SFCs demonstrated significant increases in spheroid growth compared to controls as baseline (Fig. 3). Baseline controls demonstrated modest growth with increases of $>2,000 \%$ in ES2, $278 \%$ in SKOV3, and $208 \%$ in TOV112D by day 8 . Compared to controls, all SFCs treated with an $\mathrm{Hh}$ agonist demonstrated significant 
A

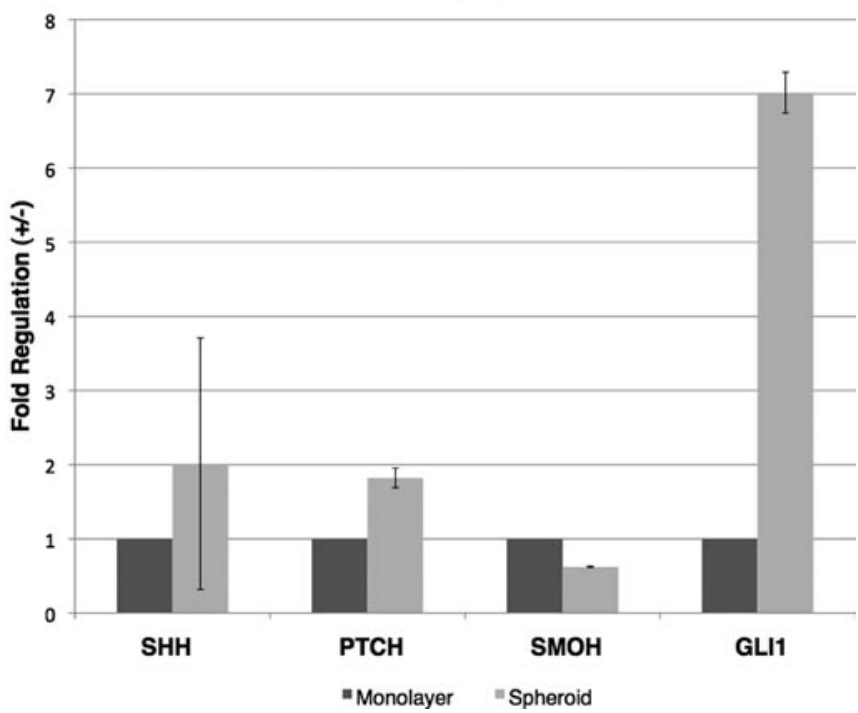

B

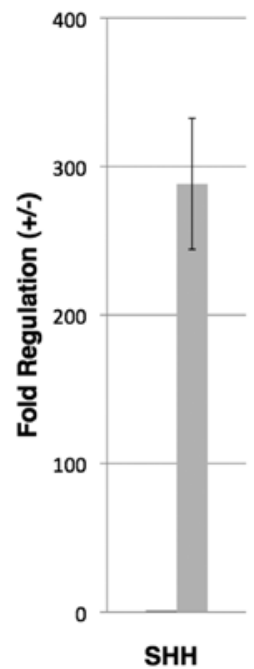

SHH

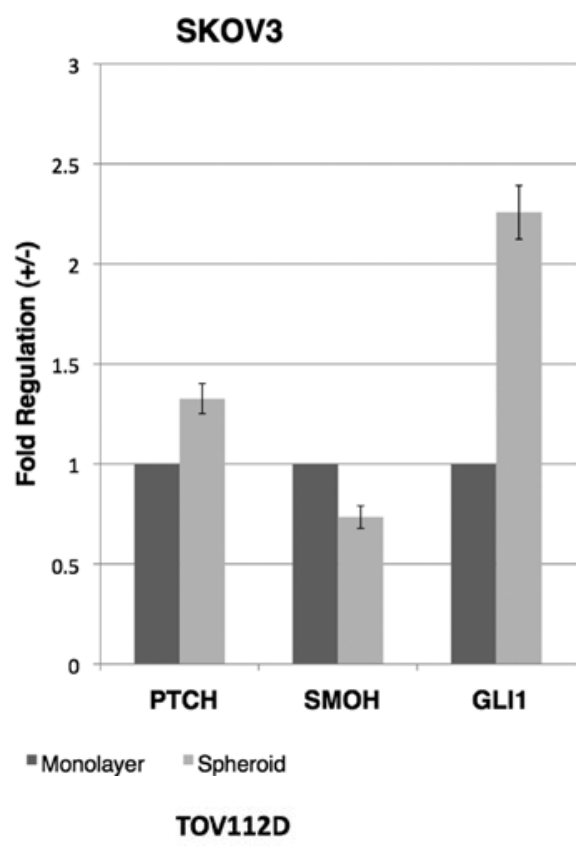

C

TOV112D

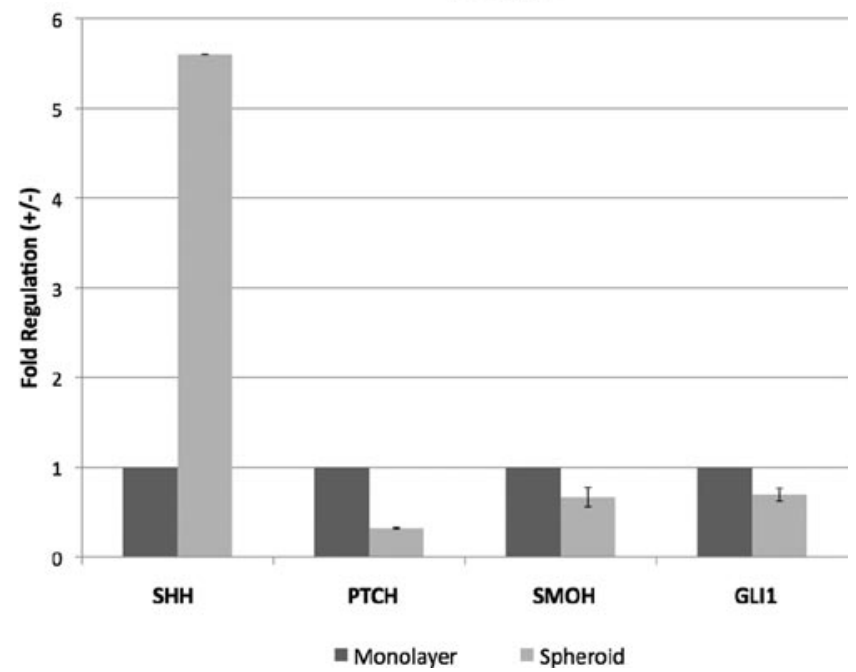

Figure 2. Hh signaling proteins: comparing monolayer to spheroid-forming cells from ovarian cancer cell lines. Total RNA was collected from monolayer and spheroid-forming cells derived from ovarian cancer cell lines (A), ES2; (B), SKOV3; and (C), TOV112D. Real-time PCR was performed using primers for SHH, PTCH, GLI1, and SMOH.
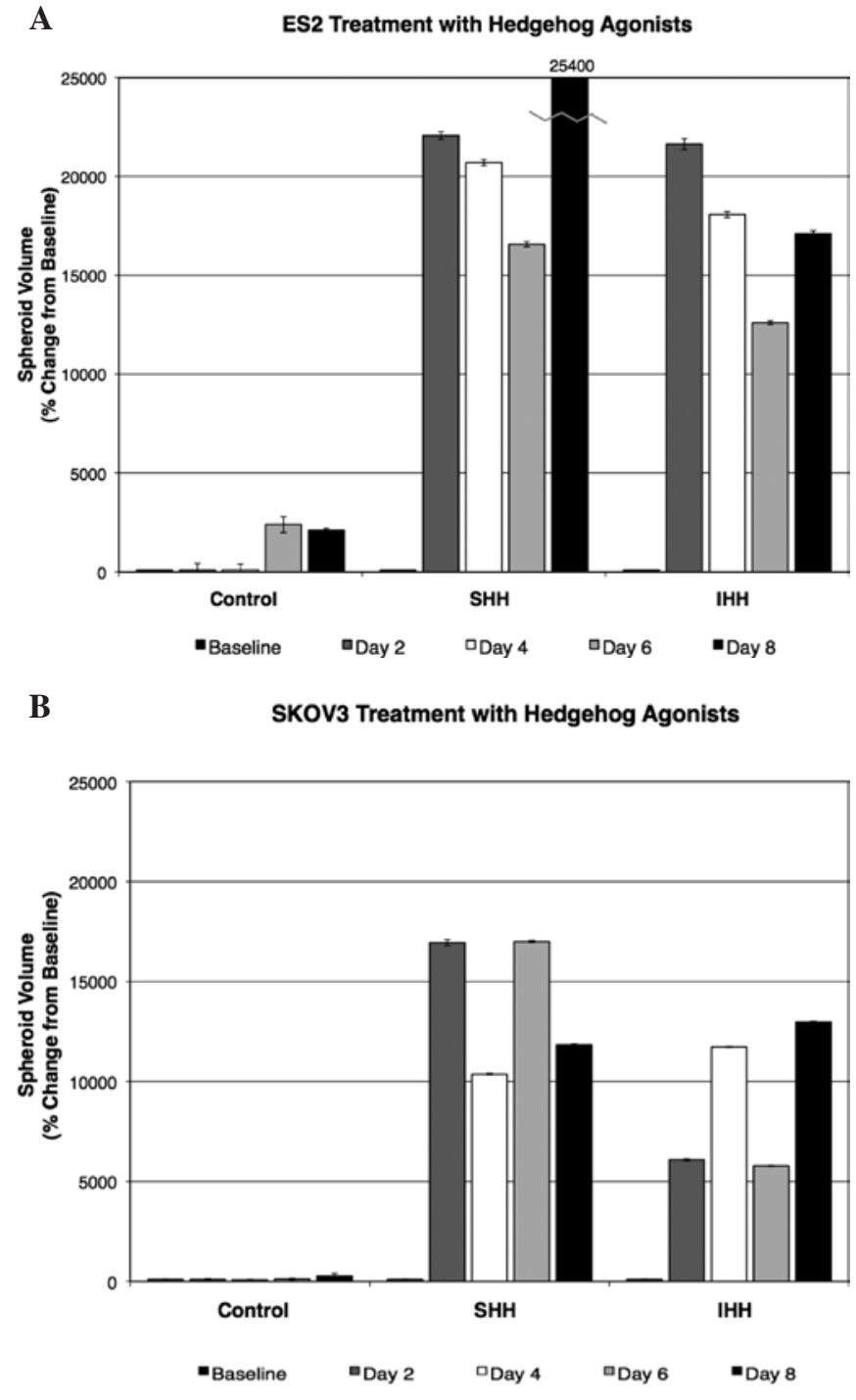

C

TOV112D Treatment with Hedgehog Agonists

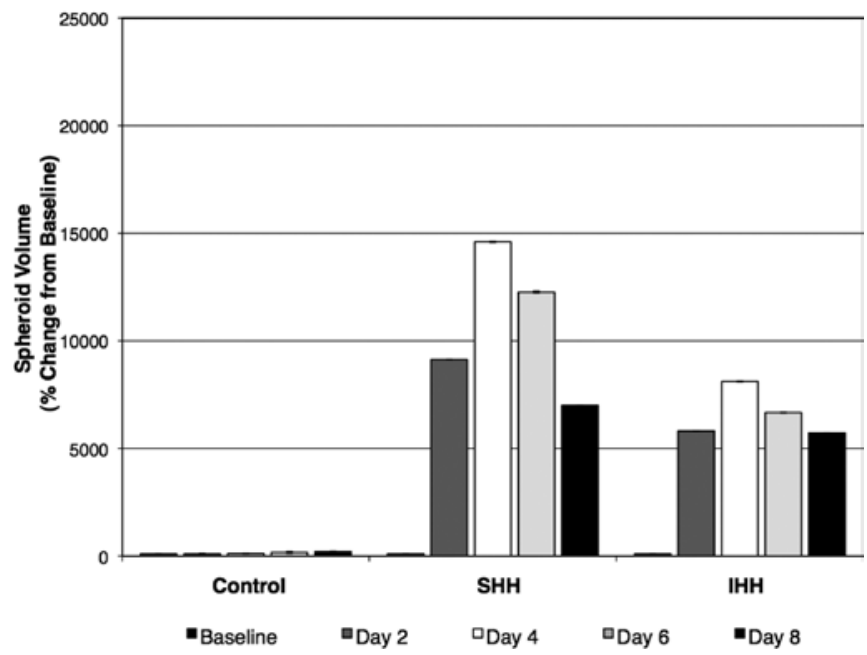

Figure 3. Activated Hh pathway significantly enhances ovarian cancer spheroid growth. Spheroid-forming cells from ovarian cancer cell lines (A), ES2; (B), SKOV3; and (C), TOV112D were treated with $\mathrm{Hh}$ agonists $\mathrm{SHH}$ and $\mathrm{IHH}$ After treatment, spheroids were monitored for 8 days with measurements taken at 2-day intervals. All ovarian cancer spheroid-forming cells demonstrated significant increases in spheroid volume compared to controls. Compared to controls (non-treatment) that showed modest growth, SHH resulted in 169-, 132-, and 220-fold increases in volume for SKOV3, TOV112, and ES2 cell lines respectively. IHH also demonstrated 143-, 74-, and 216-fold increases for same cancer cell lines. 


\section{SKOV3 Cell Lines}

DAPI
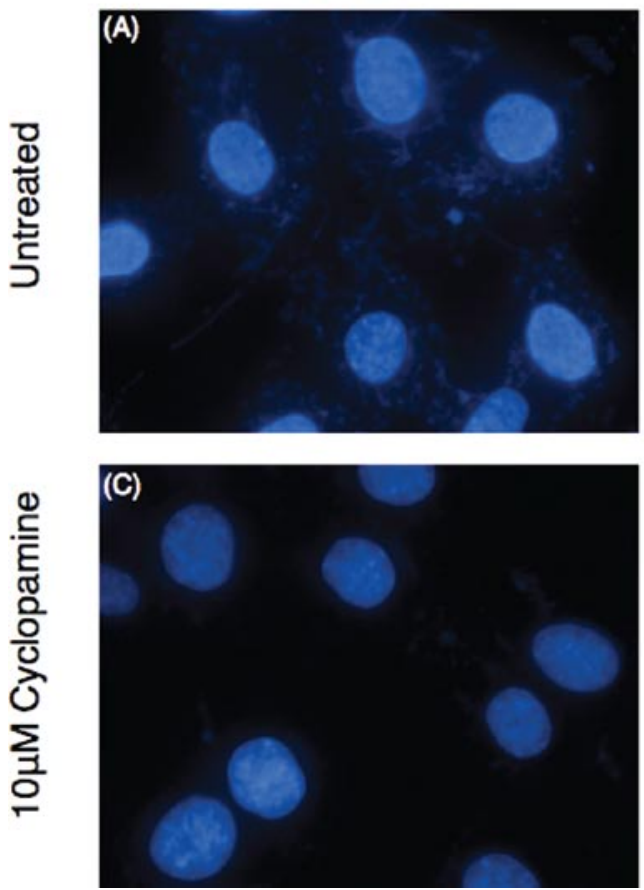

FITC
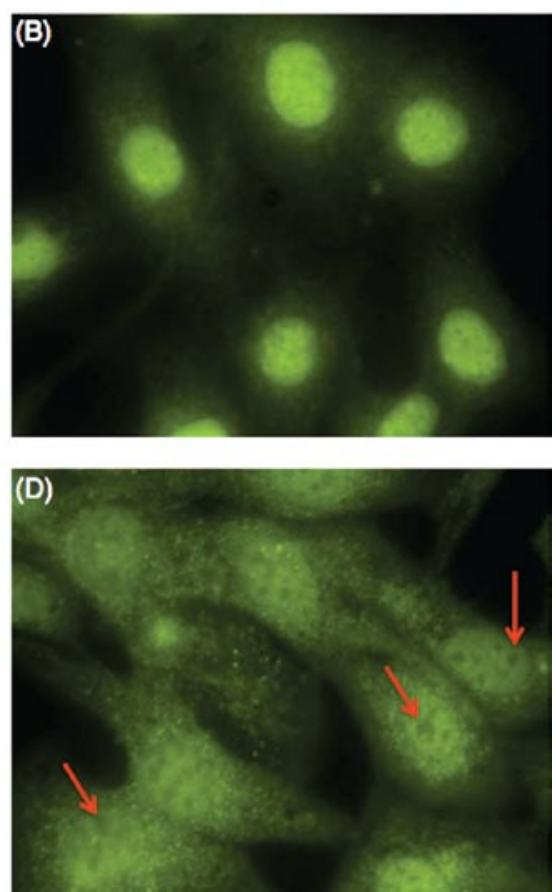

Figure 4. Cyclopamine inhibits activation of Hh pathway by preventing intranuclear accumulation of GLI1. SKOV3 ovarian cancer cells were grown and treated with LD50 concentration of cyclopamine $10 \mu \mathrm{M}$ for $24 \mathrm{~h}$. Utilizing primary antibody for GLI1 coupled with anti-IgG-FITC immunofluorescent stain, GLI1 was analyzed by microscopy for location. Intranuclear localization indicates activated Hh pathway (B and D). Nuclei were counterstained with diluted DAPI to confirm nuclei location (A and C). Without treatment, intranuclear localization of GLI1 was dominant, while cyclopamine inhibited localization of intranuclear GLI1 which resulted in uniform cellular localization with some nuclear vaculocation (red arrows).

growth. SHH-treated cells resulted in 220-, 169-, and 132-fold increased growth for ES2, SKOV3, and TOV112D respectively. Similarly, IHH demonstrated increased growth of 216-, 143-, and 74-fold.

Cyclopamine inhibits activation of Hh pathway by preventing intranuclear translocation of GLII. Utilizing immuofluorescent staining for GLI1, untreated SKOV3 cells demonstrated an abundance of intranuclear GLI1 which indicated activated $\mathrm{Hh}$ pathway. Conversely, SKOV3 ovarian cancer cells treated with LD50 concentration of cyclopamine for $24 \mathrm{~h}$ resulted in a more uniform cellular localization with evidence of nuclear vacuolization. Both indicating restriction of GLI1 from the nucleus and inhibition of Hh pathway (Fig. 4).

Inhibition of Hh pathway significantly inhibits ovarian cancer spheroid growth. SFCs derived from ovarian cancer cell lines were treated with cyclopamine (LD50 concentration) and were monitored for spheroid growth. By day 8 , the control (nontreatment) groups increased spheroid volume by $>2,000 \%$, $278 \%$, and $208 \%$ for ES2, SKOV3, and TOV112D, respectively (Fig. 5). Compared to controls, cyclopamine reduced spheroid growth: 10-fold in ES2, 5-fold in SKOV3, and 4-fold in TOV112D cell lines.

Ovarian cancer spheroid-forming cells are more resistant to cyclopamine than monolayer. Spheroids derived from ovarian cancer cell lines ES2, SKOV3, and TOV112D were treated with the determined LD50 concentrations of cyclopamine. Compared to monolayer ovarian cancer cells, SFCs demonstrated increase in resistance to cyclopamine with an approximate 50\%, 30\% and $30 \%$ improvement in survival for ES2, SKOV3, and TOV112D, respectively (Fig. 6). Of note, RT-PCR demonstrated that $\mathrm{SMOH}$ receptor was down-regulated in SFCs compared to monolayer (Fig. 2) and represents a likely cause of the resistance seen in SFCs.

\section{Discussion}

Cancer derived spheroid-forming cells have been shown to display cancer stem cells properties of self-renewal cells $(5,17)$ and chemoresistance (18). Although CSCs comprise a small percentage of the total cancer cells within a tumor, they are considered to be solely responsible for cancer self-renewal, invasion, metastasis, and resistance to therapy $(15,16)$. Thus, detecting innate molecular pathways important in CSCs could give further insight towards targeted therapy and improvements in treatment.

Our report evaluates the hedgehog pathway considering it has shown considerable involvement in cancers. This signal transduction pathway controls cancer processes such as proliferation, differentiation and organogenesis and has a rationale in CSCs. Similar to others who found hedgehog signaling pathway to be activated in ovarian cancer (11), we demonstrated the increased expression of intranuclear GLI1 in all ovarian cancer cell lines compared to normal immor- 
A

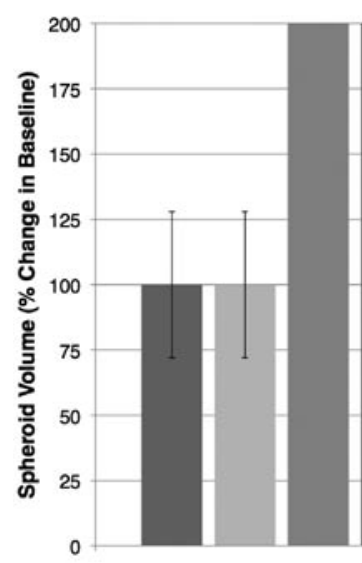

Control

"Day 2 "Day 4 "Day 6 "Day 8

$\mathbf{B}$

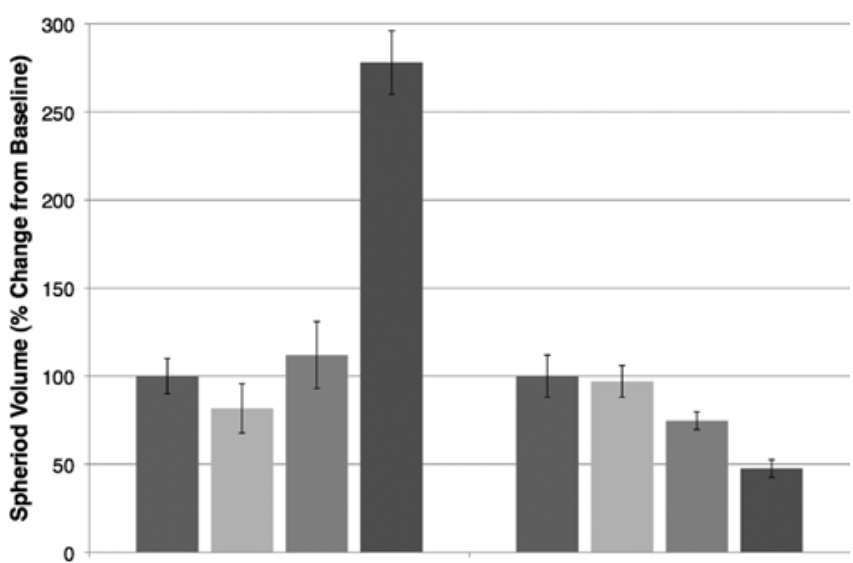

Control Cyclopamine

-Day 2 "Day 4 "Day 6 -Day 8

C

TOV112D

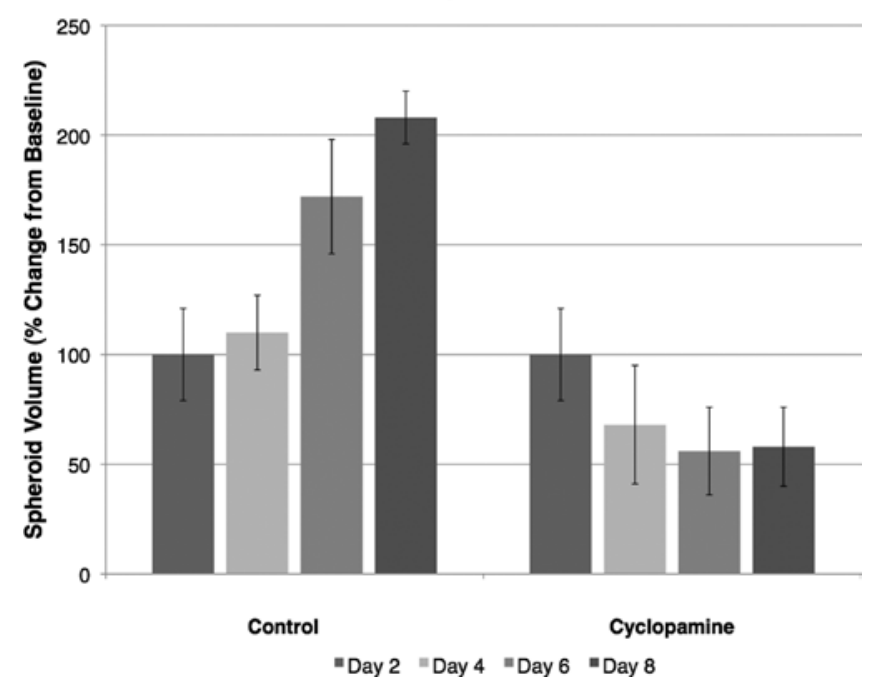

Figure 5. Inhibition of Hh pathway significantly inhibits ovarian cancer spheroid growth. Spheroid-forming cells derived from (A), ES2; (B), SKOV3; and (C), TOV112D were treated with cyclopamine, a SMOH receptor inhibitor. SFCs were monitored for growth in similar fashion to Fig. 3. Spheroid growth was signficantly halted with cyclopamine when compared to non-treated controls, with a 10-, 5-, and 4-fold reduction in day 8 volume in (A), ES2; (B), SKOV3; and (C), TOV112D cell lines, respectively.
A

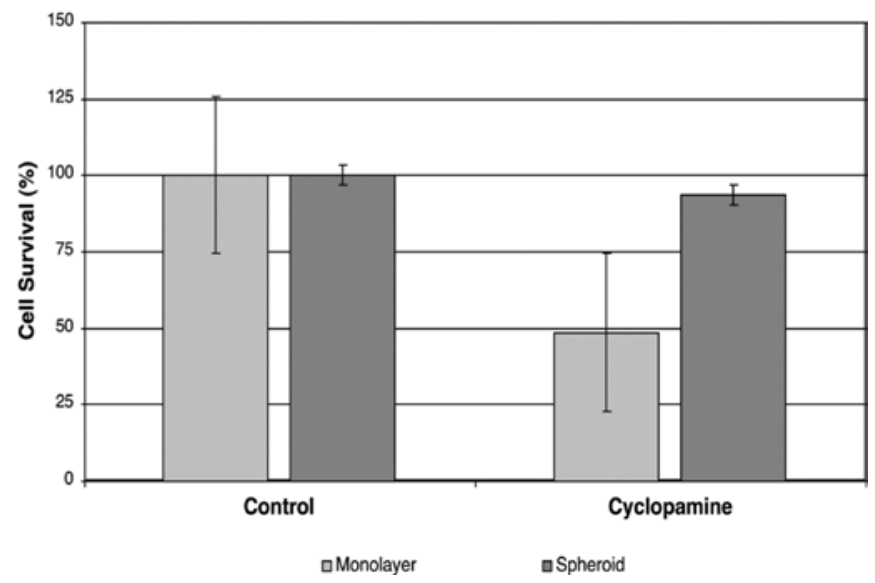

B

SKOV3

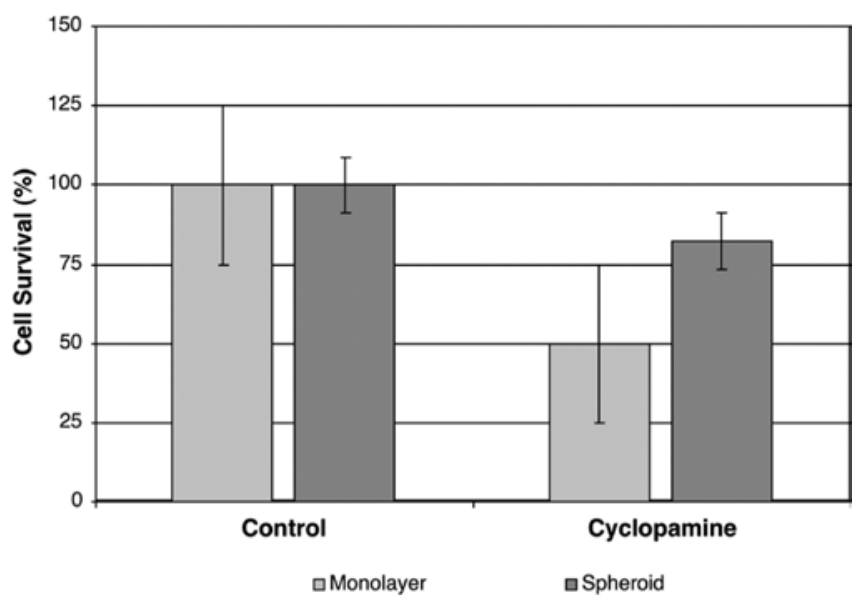

C

TOV112D

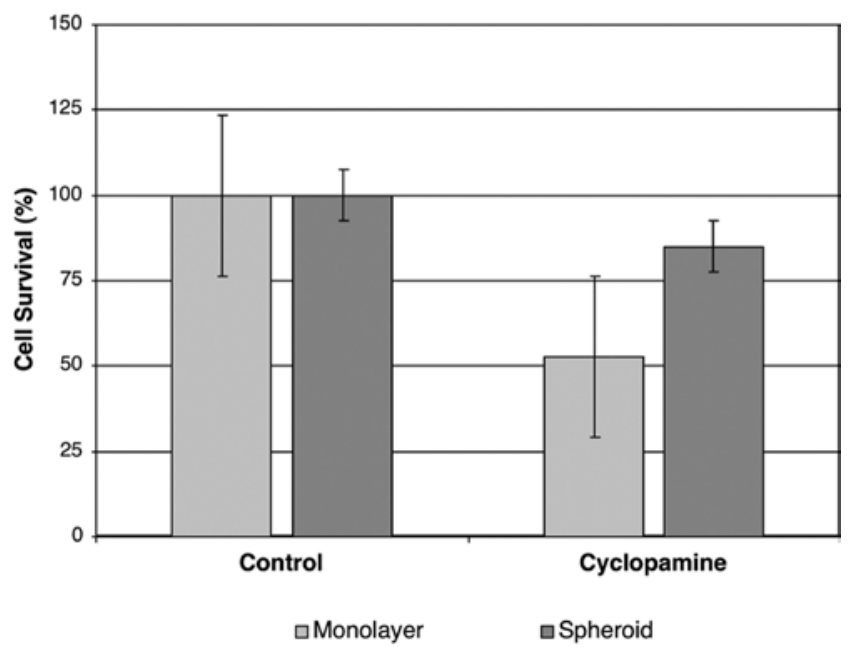

Figure 6. Ovarian cancer spheroid-forming cells are significantly more resistant to cyclopamine than monolayer. The LD50 concentration of cyclopamine was determined for each cell line. LD50 was used to treat both monolayer and SFCs from (A), ES2; (B), SKOV3; and (C), TOV112D ovarian cancer cell lines. Using MTS assay, survival at $24 \mathrm{~h}$ in monolayer and SFCs was calculated. Compared to monolayer, SFCs demonstrated increased resistance to cyclopamine with a $50 \%, 30 \%$, and $30 \%$ increase in survival for (A), ES2; (B), SKOV3; and (C), TOV112D, respectively. 
talized ovarian cells (IOSE80). Other Hh signaling molecules (SMOH and PTCH) were seen in varying levels of expression in all cell lines evaluated. Similarly, Chen et al (11) demonstrated that normal epithelium, benign ovarian tumor, borderline ovarian tumor, and ovarian cancer all possessed $\mathrm{Hh}$ related proteins at varying concentrations. These findings are to be expected considering $\mathrm{Hh}$ is a normally dormant molecular pathway innate to most cells.

Considering the role of the Hh pathway in proliferation, differentiation and organogenesis, we described the affect of growth of SFCs in ovarian cancer with the upregulation and down-regulation of this pathway. Using $\mathrm{Hh}$ agonists, $\mathrm{SHH}$ and IHH, significantly increased growth in all ovarian cancer spheroid-forming cells evaluated. This growth, based on spheroid volume, averaged an increase of $\sim 15,000 \%$ for $\mathrm{SHH}$ and $\sim 10,000 \%$ for IHH treated spheroids over baseline controls. This effect was more pronounced than a similar report by Liu et al who reported that $\mathrm{SHH}$ and $\mathrm{IHH}$ treated primary mammospheres formed $100 \%$ more secondary mammospheres and the cell numbers per secondary mammosphere were increased (19).

Conversely, inhibition of this pathway via a SMOH inhibitor, cyclopamine, showed a significant reduction in spheroid volume. We also determined that ovarian cancer SFCs maintained the stem-cell like property $(20,21)$ of resistance to cyclopamine therapy compared to monolayer. Despite having a higher percentage of cyclopamine treated cells surviving in SFCs, all showed a significant reduction in spheroid volume compared to control.

This corresponds to the known role of Hh pathway controlling the developmental cancer processes (carcinogenesis, tumor proliferation, differentiation and self-renewal). This affect appears to be achieved by preventing the translocation of GLI1 into the nucleus as depicted by our immunofluorescent localization of GLI1. The apparent levels of GLI1 was not affected, but was simply excluded from the nucleus with nuclear shadowing and vacuolization.

Interestingly, SFCs from cancers cell lines showed innate increases in $\mathrm{SHH}$ at baseline. Once treated with either $\mathrm{Hh}$ agonist, $\mathrm{SHH}$ or $\mathrm{IHH}$, significant increases in spheroid growth and volume was seen. Additionally, SFCs in 3 of 4 cell lines evaluated demonstrated down-regulation of $\mathrm{SMOH}$ receptor. Our Hh antagonist, cyclopamine, is a $\mathrm{SMOH}$-inhibitor and therefore the resistance in SFCs could partly be due to less SMOH available in SFCs. However, despite a down-regulation of $\mathrm{SMOH}$, growth was still halted significantly with cyclopamine. One could surmise from these data combined with the known functions of the Hh pathway, that Hh-inhibition might be more important in disrupting the growth of cancer and less important in cell death and/or survival. The studies described within cannot completely address these issues, however, the potential to inhibit further growth of cancer might serve as an important adjunct to cytotoxic chemotherapy in the clinical arena.

The Hh pathway appears to be important in regulating the growth of ovarian cancer SFCs in vitro. These SFCs are reported to contain the properties of cancer stem cells of self-renewal, differentiation, and chemoresistance, and as such they represent the most aggressive cancer cells. In these cells, the activation of $\mathrm{Hh}$ pathway with $\mathrm{Hh}$ agonists correlated to significantly enhanced growth, while inhibition with the SMOH-inhibitor cyclopamine resulted in significant growth restriction. This regulation on ovarian cancer growth warrants further evaluation in ovarian cancer.

\section{Acknowledgements}

This study was supported by the Abraham A. Mitchell Clinical Cancer Research Scholarship.

\section{References}

1. Jemal A, Siegel R, Ward E, Hao Y, Xu J and Thun MJ: Cancer statistics. CA Cancer J Clin 59: 225-249, 2009.

2. Ozols RF, Bundy BN, Greer BE, Fowler JM, Clarke-Pearson D, Burger RA, Mannel RS, De Geest K, Hartenbach EM and Baergen R: Phase III trial of carboplatin and paclitaxel compared with cisplatin and paclitaxel in patients with optimally resected stage III ovarian cancer: a Gynecologic Oncology Group study. J Clin Oncol 21: 3194-3200, 2003.

3. Jordan CT, Guzman ML and Noble M: Cancer stem cells. N Engl J Med 355: 1253-1261, 2006.

4. Alvero AB, Chen R, Fu HH, Montagna M, Schwartz PE, Rutherford T, Silasi DA, Steffensen KD, Waldstrom M, Visintin I and Mor G: Molecular phenotyping of human ovarian cancer stem cells unravels the mechanisms for repair and chemoresistance. Cell Cycle 8: 158-166, 2009.

5. Zhang S, Balch C, Chan MW, Lai HC, Matei D, Schilder JM, Yan PS, Huang TH and Nephew KP: Identification and characterization of ovarian cancer-initiating cells from primary human tumors. Cancer Res 68: 4311-4320, 2008.

6. Al-Hajj M, Wicha MS, Benito-Hernandez A, Morrison SJ and Clarke MF: Prospective identification of tumorigenic breast cancer cells. Proc Natl Acad Sci USA 100: 3983-3988, 2003.

7. Azoulay S, Terry S, Chimingqi M, Sirab N, Faucon H, Gil Diez de Medina S, Moutereau S, Maille P, Soyeux P, Abbou C, Salomon L, Vacherot F, De la Taille A, Loric S and Allory Y: Comparative expression of Hedgehog ligands at different stages of prostate carcinoma progression. J Pathol 216: 460-470, 2008.

8. Kubo M, Nakamura M, Tasaki A, Yamanaka N, Nakashima H, Nomura M, Kuroki S and Katano M: Hedgehog signaling pathway is a new therapeutic target for patients with breast cancer. Cancer Res 64: 6071-6074, 2004.

9. Liao X, Siu MK, Au CW, Chan QK, Chan HY, Wong ES, Ip PP, Ngan HY and Cheung AN: Aberrant activation of hedgehog signaling pathway contributes to endometrial carcinogenesis through beta-catenin. Mod Pathol 22: 839-847, 2009.

10. Das S, Harris LG, Metge BJ, Liu S, Riker AI, Samant RS and Shevde LA: The hedgehog pathway transcription factor GLI1 promotes malignant behavior of cancer cells by up-regulating osteopontin. J Biol Chem 284: 22888-22897, 2009.

11. Chen JK, Taipale J, Cooper MK and Beachy PA: Inhibition of Hedgehog signaling by direct binding of cyclopamine to Smoothened. Genes Dev 16: 2743-2748, 2002.

12. Berman DM, Karhadkar SS, Maitra A, Montes de Oca R, Gerstenblith MR, Briggs K, Parker AR, Shimada Y, Eshleman JR, Watkins DN and Beachy PA: Widespread requirement for Hedgehog ligand stimulation in growth of digestive tract tumours. Nature 425: 846-851, 2003.

13. Thayer SP, Di Magliano MP, Heiser PW, Nielsen CM, Roberts DJ, Lauwers GY, Qi YP, Gysin S, Fernandez-del Castillo C, Yajnik V, Antoniu B, McMahon M, Warshaw AL and Hebrok M: Hedgehog is an early and late mediator of pancreatic cancer tumorigenesis. Nature 425: 851-856, 2003.

14. Fujiwara T, Grimm EA, Mukhopadhyay T, Cai DW, OwenSchaub LB and Roth JA: A retroviral wild-type p53 expression vector penetrates human lung cancer spheroids and inhibits growth by inducing apoptosis. Cancer Res 53: 4129-4133, 1993.

15. Rich JN: Cancer stem cells in radiation resistance. Cancer Res 67: 8980-8984, 2007.

16. Mimeault M, Hauke R, Mehta PP and Batra SK: Recent advances in cancer stem/progenitor cell research: therapeutic implications for overcoming resistance to the most aggressive cancers. J Cell Mol Med 11: 981-1011, 2007. 
17. Seaberg RM and van der Kooy D: Stem and progenitor cells: the premature desertion of rigorous definitions. Trends Neurosci 26 : $125-131,2003$.

18. Wani AA, Sharma N, Shouche YS and Bapat SA: Nuclearmitochondrial genomic profiling reveals a pattern of evolution in epithelial ovarian tumor stem cells. Oncogene 25: 6336-6344, 2006.

19. Liu S, Dontu G, Mantle ID, Patel S, Ahn NS, Jackson KW, Suri P and Wicha MS: Hedgehog signaling and Bmi-1 regulate selfrenewal of normal and malignant human mammary stem cells. Cancer Res 66: 6063-6071, 2006.

20. Dean M, Fojo T and Bates S: Tumour stem cells and drug resistance. Nat Rev Cancer 5: 275-284, 2005.
21. Frank NY, Margaryan A, Huang Y, Schatton T, WaagaGasser AM, Gasser M, Sayegh MH, Sadee W and Frank MH: ABCB5-mediated doxorubicin transport and chemoresistance in human malignant melanoma. Cancer Res 65: 4320-4333, 2005.

22. Sadowski HB, Shuai K, Darnell JE Jr and Gilman MZ: A common nuclear signal transduction pathway activated by growth factor and cytokine receptors. Science 261: 1739-1744, 1993. 